\title{
ZAHNWALFUNDE IN SCHWEDEN
}

\author{
von \\ JOHANNES LEPIKSAAR \\ Naturhistoriska Museet, Göteborg
}

Daß eine Tiergruppe pelagischer Anpassung, wie die Zahnwale, in den mit dem Ozean nur mittelbar, via Nordsee, in Verbindung stehenden Küstenmeeren Schwedens eine weit bescheidenere Rolle spielt als an der atlantischen Küste Europas, ist natürlich und vorauszusetzen. Von 23 europäischen Arten sind für Schweden bis jetzt nur 14, die meisten als seltene Irrgäste, festgestellt worden. Desto mehr man sich dabei längs der Küste von der Nordsee entfernt, desto geringer wird die Anzahl. Schon an der Westküste sinkt sie von 14 im Skagerrak bis nur 9 Arten im Kattegatt. In der Ostsee hat man an der Südküste zuerst noch 8, an der Ostküste 4 und im Bottnischen Meerbusen endlich nur 2 (Abb. 1).

Als heimische, zu allen Jahreszeiten öfters antreffbare Bewohner kann man für die Westküste nur zwei Arten nennen - den ziemlich häufigen Schweinswal (Phocaena phocoena) und den weit selteneren Langfinnendelphin (Lagenorhynchus albirostris). Das große Brackwassergebiet der Ostsee hat nur den Schweinswal allein heimisch. Der größte Teil seines Bestandes verläßt jedoch in der Regel dieses Gebiet für die Winterzeit. Seine Wanderzüge von und zu der Ostsee sind den Küstenbewohnern, besonders der Oresundgegend und der Südküste seit altem wohlbekannt und wurden an dazu geeigneten Stellen, wie Ravlunda an der Ostküste von Schonen (LinNaEus, 1751) oder Torekov und die Laholmbucht im Südostteil des Kattegattes (NILsson, 1847), noch im vorigen Jahrhundert zum Fang dieser Kleinwale ausgenutzt (Abb. 2). Im Gegensatz zu der herbstlichen Schweinswalsjagd in den dänischen Sunden geschah dies hier hauptsächlich während der Früjahrwanderung der Tiere.

Es ist bestimmt kein Zufall, daß die beiden hier für Schweden als heimisch bezeichneten Zahnwale auch sonst als litorale Formen aufzutreten pflegen und daß sie sich auch morphologisch von ihren pelagischen Verwandten durch das Fehlen oder schwä- chere Ausbildung spezieller Anpassungen, wie die Kielbildungen der Schwanzwurzel, unterscheiden. $\mathrm{Da} ß$ sie sich an den schwedischen Küsten wirklich heimisch fühlen, geht auch aus der Tatsache hervor, daß bei diesen zwei Arten die aktiven Strandungen nur selten vorkommen. Den Schweinswal und den Langfinnendelphin pflegt man meistens durch Feldbeobachtungen freischwimmender Tiere, durch Funde der in Fischgeräte geratenen Individuen oder auf Grund der passiven Strandungen von Kadavern und Skelett-teilen der irgendwie irgendwo verunglückten Exemplare, festzustellen. Öfters scheint es sich dabei entweder um von der Eisbildung überraschten und unter der Eisdecke erstickten Individuen oder von Fahrzeugen beschädigten Tieren zu handeln. Ein Massensterben von Schweinswalen infolge des frühzeitigen Zufrierens der Sunde und Buchten ist wiederholt bei Gotland und an den Küsten von Schonen festgestellt worden (Fauna och Flora, 1940; EKMAN, 1949; SvÄrDSON, 1957). Zufällig kann ähnliches auch mit dem Langfinnendelphin an der Westküste der Fall sein, wie z.B. im strengen Winter 1940/41 (BerLin, 1941; Hanström, 1950).

Ein ziemlich regelmäßiger Besucher der Westküste ist der Schwertwal (Orcinus orca). Von diesem räuberischen Kosmopoliten der Weltmeere hat man seit dem vorigen Jahrhundert 12 Funde von der Westküste und wenigstens 6 aus den Südteil der Ostküste Schwedens.

Die übrigen Zahnwalarten sind alle. viel seltener und unregelmäßiger angetroffen worden. Die meisten Funde dieser Irrgäste stammen aus den Herbstmonaten August und September oder April-Mai im Frühling. Von Januar und Februar wie auch vom Juli sind bisher keine bekannt.

Die seltensten sind die nichtpolaren Kosmopoliten und Nahrungsspezialisten-Cephalopodenfresser wie der Cuvier-Schnabelwal (Ziphius cavirostris), der Rundkopfdelphin (Grampus griseus), der Grindwal 


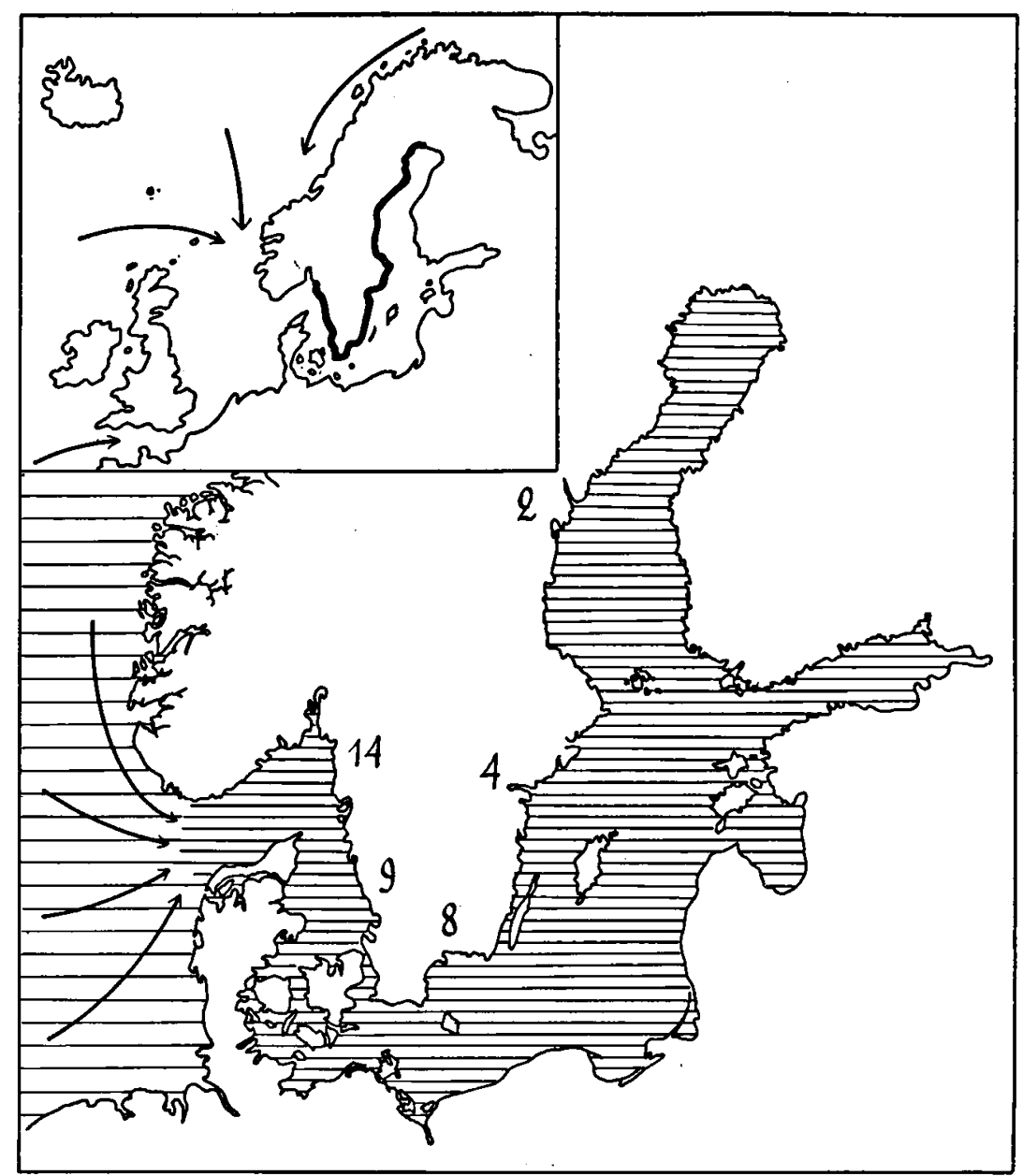

Abb. 1. Kleine Karte: Die Kïstenlinie Schwedens mit fetter Linie markiert. Große Karte: Das „Reusensystem " vom Skagerrak bis Ostsee (dichte Schraffur). Die an der Kiistenlinie angebrachten Ziffer geben die Anzahl der angetroffenen Zahnwalarten für betreffenden Küstengebiet an.

(Globicephala melaena) und der Kleinschwertwal (Pseudorca crassidens) (Abb. 3). Mit Ausnahme des letztgenannten, von welcher Art die im Jahre 1861 in die Nordsee eingedrungene Herde mehrere Strandungen sowohl an der West- wie Südküste hinterlassen hat, sind die Funde dieser Gruppe Einzelfunde und auf die Skagerrakküste begrenzt. Der Rundkopfdelphin ist dort einmal, die übrigen zweimal entdeckt worden. $\mathrm{Zu}$ dieser Gruppe der Irrgäste läßt sich am besten auch der bisher einzige Fund des Pottwales (Physeter macrocephalus) der historischen Zeit, nämlich 1718, anschließen (BERnströM, 1949).

Von den piscivoren Arten derselber Verbreitungsgruppe (Abb. 4) ist der Großtümmler (Tursiops truncatus) der häufigste. Er ist wenigstens $5 \mathrm{mal}$ an der Westkuiste angetroffen worden. Den sogenannten ge- meinen Delphin (Delphinus delphis) ist man 3mal an der West- und einmal an der Ostküste begegnet.

Von den nordatlantischen Zahnwalen (Abb. 5) ist der Sowerby-Zweizahnwal (Mesoplodon bidens) der häufigste. Er hat $4 \mathrm{mal}$ an der Westküste und einmal am Öresund gestrandet. Vom Weiß-seitendelphin (Lagenorhynchus acutus) besitzt man 4 sichere Funde an der Westküste, vom Dögling (Hyperoodon ampullatus) 2 sichere für die Westküste, 1 vom Öresund und einen von der Ostküste.

Eine besondere Stellung nimmt das Vorkommen des arktischen Weißwales (Delphinapterus leucas) ein. In den schwedischen Küstengewässern ist man ihm bisher nur 2 mal begegnet. Im 1841 soll ein weißer Wal bei Töre in Norrbotten angetroffen sein (MALM, 1877) und im 1964 wurden 1-3 Tiere wiederholt von 


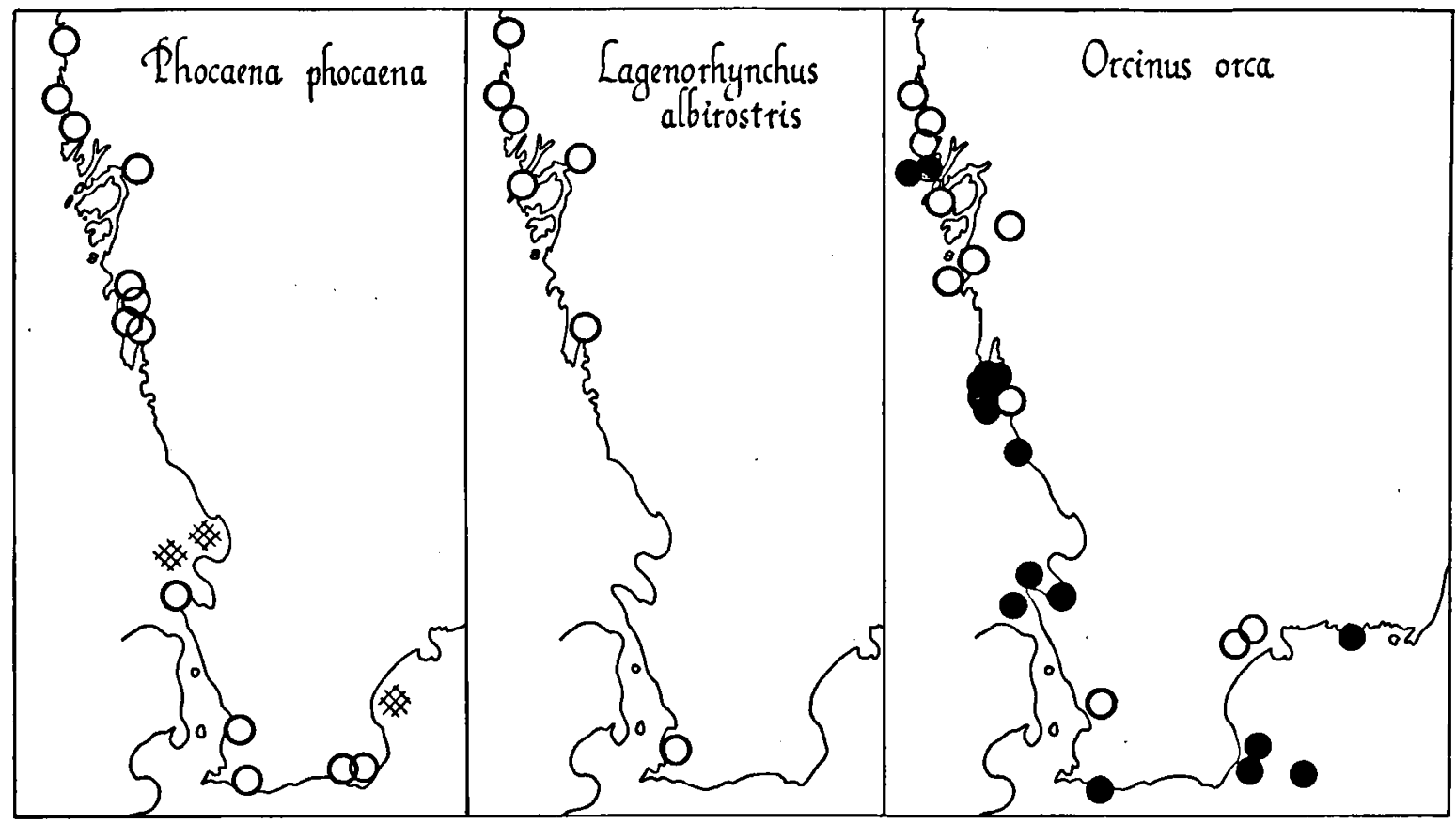

Abb. 2. Verteilung der Fundorte des Schweinswales, des Langfinnendelphins und des Schwertwales. Offene Kreise subfossiles Vorkommen, schwarze Punkte - rezentes Auftreten. Die Gitterfiguren links markieren die Plätze des Schweinswalfanges im 18. und 19. Jh. Die Namen der Fundorte werden von links oben der Küstenlinie entlang aufgezählt.

Phocaena phocoena: Dafter, Otterö, Rörvik, Bräcke, Gamlestaden, Fagelsten, Kyvik, Gröninge, Lahebiahöhle, Arlöv, Dalköpinge, Valleberga, Hagestad. "

Lagenorhynchus albirostris: Dafter, Otterö, Rörvik, Nedre Kuröd, Ramholm, Gröninge, Hötofta.

Orcinus orca: Otterö, Rörvik, Sotenkanal, Lysekil (2 Funde), Rödmyr, Gamla Lödöse, Castellgården-Ytterby, Rörö, die Anhäufung der rezenten Funde in der Mitte der Karte umfaßt Frillesås, Brattö, Vändelsö und Balgö, dann folgen Varberg (subfossiles Vorkommen), Akerös, Vegeholm, Kulla-Leuchtturm, Kullen, Lund (Erdfund), Trelleborg, Simrishamn (2 Funde), Bornholm, Ormtofta, Ivö, Ronneby.

Mai bis September sowohl in der Ostsee wie auch an der Westküste beobachtet, photographiert und zuletzt auch eines an der Skagerrakküste tot angeschwemmt aufgefunden. Es scheint, als ob eine Herde dieser Art in das Skagerrak-Ostsee-System eingedrungen und nach einer Zeit des Herumirrens sich in kleinere Verbände zersprengt und zerstreut hätte. Der Weißwal pflegt bekanntlich, wie viele andere Arten der klimatisch instabilen arktischen Zone, sporadisch als Invasionsgast südlich seines eigentlichen Lebensraumes aufzutreten. $\mathrm{Da}$ er dabei gelegentlich seine Fluchtzüge bis in die Nordsee und von dort via Skagerrak-Kattegatt-Öresund oder die dänischen Sunde bis nach den Bottnischen und Finnischen Meerbusen der Ostsee erweitert, ist neben den schwedischen auch durch Funde aus Finnland, Estland, Litauen und Dänemark für die Jahre 1869, $1903,1904,1906-1908$ und 1964 belegt worden (Lilljeborg, 1874; Winge, 1904; Japha, 1908; GrevE, 1909). (Abb. 6.)
Das Wassersystem vom Skagerrak bis zur Ostsee scheint für die Zahnwale, ebenso wie für viele andere nektonische Formen der ozeanischen Fauna, eine Naturfalle, durch das Fischreichtum seines Mündungsgebietes wie geköderte Riesenreuse darzustellen. Die einmal in diese Falle hineingeratenen oder eingelockten pelagischen Waltiere scheinen dort in den relativ schmalen Passagen und vielen Schärenhöfen ihre Orientierung zu verlieren und stranden nach einer kürzeren oder längeren Zeit des Herumirrens in der Regel an einer der lauernden Untiefen. Diese Naturfalle muß seit der Hochwärmezeit, d.h. rund 7000 Jahren bestanden haben, meist effektiv jedoch erst in den letzten Jahrtausenden wo nach der litorinazeitlichen Transgressionen die Passagen zwischen den Einzelabteilungen dieses komplexen Reusensystems schmäler als unter der Wärmezeit geworden sind.

9 Zahnwalarten haben ihre subfossilen Reste in Schweden hinterlassen, die auf zeitliche Veränderun- 


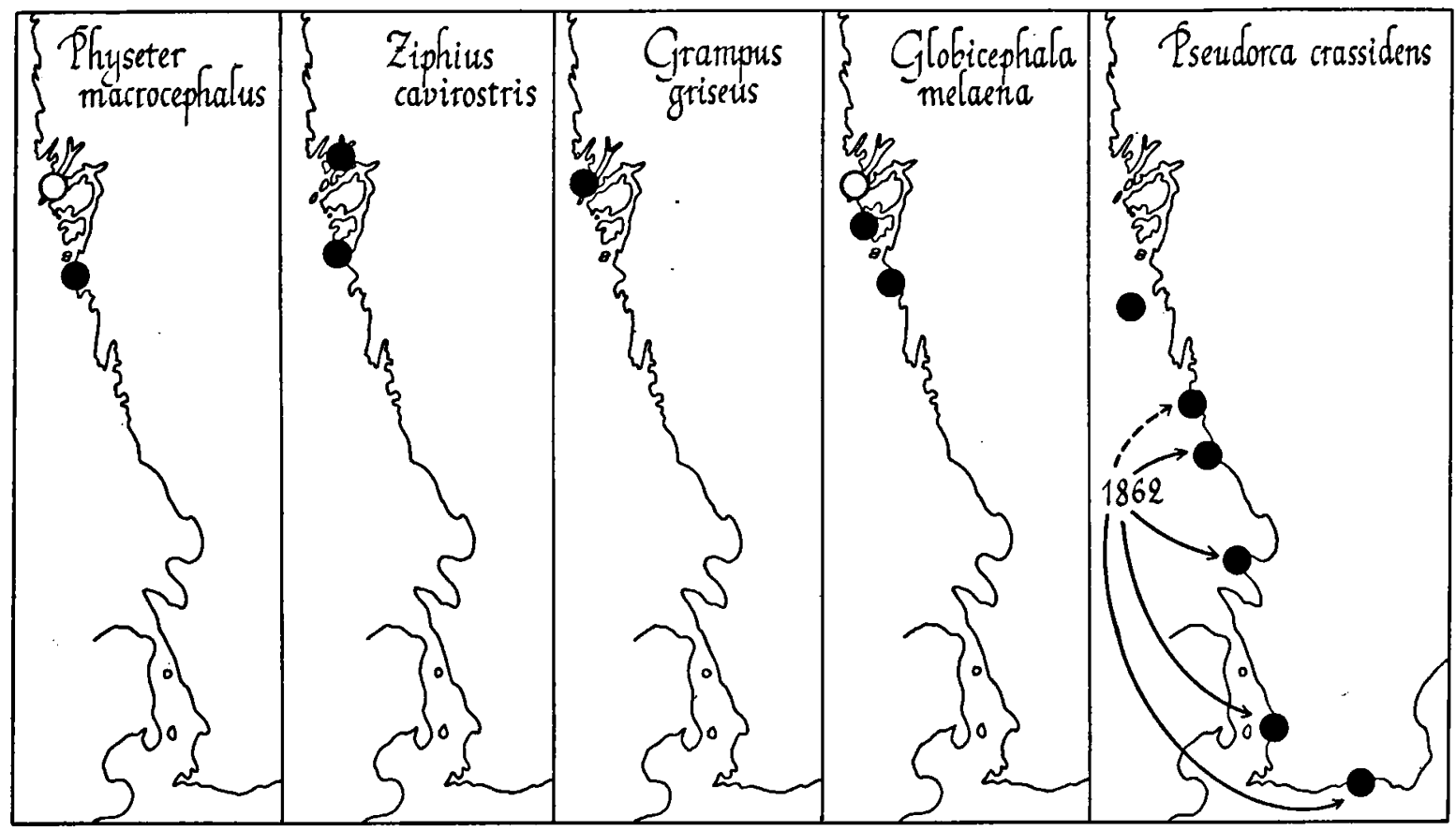

Abb. 3. Fundorte der cephalopodenfressenden Kosmopoliten: des Pottwales, des Cuvier-Schnabelwales, des Rundkoptdelphines, des Grindwales und des Kleinschwertwales.

Physeter macrocephalus: Backa, Overö. Erdfund von Värmdö bei Stockholm an der Ostküste weggelassen.

Ziphius cavirostris: Holma, Marstrand.

Grampus griseus: Gåsö.

Globicephala melaena: Värbyfiord, Tiörn, Biörlanda.

Pseudorca crassidens: Vinga, Varberg, Falkenberg, Torekov, Sege-Fluss, Ystad.

gen quali- und quantitativer Art hinweisen.

Die ältesten Reste gehören dem arktischen Weißwal an. 22 Funde (Abb. 6, Detailkarte), meist Skelettfunde, hat man bisher aus dem vom spätglazialen und vorwärmezeitlichen Meer überfluteten Teil Westschwedens angetroffen. Es ist merkwürdig, daß uns bisher keine Funde des in dem gegenwärtigen Eismeer vorkommenden Schwertwales vorliegen die man den Ablagerungen des spätglazialen Meeres zuschreiben könnte (diejenigen Zahnfunde die RingSTRÖM (1928) aus den Schalenbänken von Uddevalla angibt sind nach NyBeLIN (1940) fehlbestimmt). Man weiß auch nicht bestimmt ob in diesem, nordwärts an den weichenden Eisrand angrenzenden Meeresteil, der hocharktische Narwal (Monodon monoceros) vorgekommen ist. Zwei Funde sind angemeldet jedoch ist ihre Herkunft nicht genügend klargestellt.

In den Schalenablagerungen der Uddevalla-Gegend (Schalenbänke bei Kuröd und Bräcke) an der Westküste, die hauptsächlich während der Vorwärmezeit abgelagert sind (ODHNER, 1930; Bృörsjö, 1953), dominieren noch Weißwalreste (RINGström, 1928). Daneben sind auch einzelne Funde des Schweins- wales und Langfinnendelphins entdeckt worden. Die letzteren Arten müssen als Zeugen des beginnenden atlantischen Einflußes aufgefaßt werden.

Ich möchte hierbei hervorheben, $\mathrm{da} B$ bis jetzt keine Zahnwalfunde aus dem vorwärmezeitlichen mittelschwedischen Sunde zwischen Westmeer und YoldiaMeer des Ostseebeckens angetroffen worden sind. Auch fehlen aus der Ostsee selbst bisher jegliche Funde, bis man erst in den frühatlantisch-mesolithischen Küchenabfällen der Kunda-Siedlung in Nordestland Schweinswal begegnet, welche Art offensichtlich erst durch die neue südliche Verbindung zwischen Nord- und Ostsee eingewandert ist.

Durch diese neue Pforte sind in das Litorina-Meer des Ostseebeckens zur Wärmezeit auch die mehr pelagischen Vertreter der nichtarktischen Zahnwale eingedrungen, wie wenigstens zwei Funde des Großtümmlers (einer bei Tosterup an der Südküste, der zweite sogar so nördlich wie bei Sundsvall am Bottnischen Meerbussen) beweisen. Diese Art ist wahrscheinlich in der Wärmezeit ein viel häufiger Besucher der schwedischen Meere gewesen als gegenwärtig, wo er nur als Irrgast zu bezeichnen ist. Viel- 


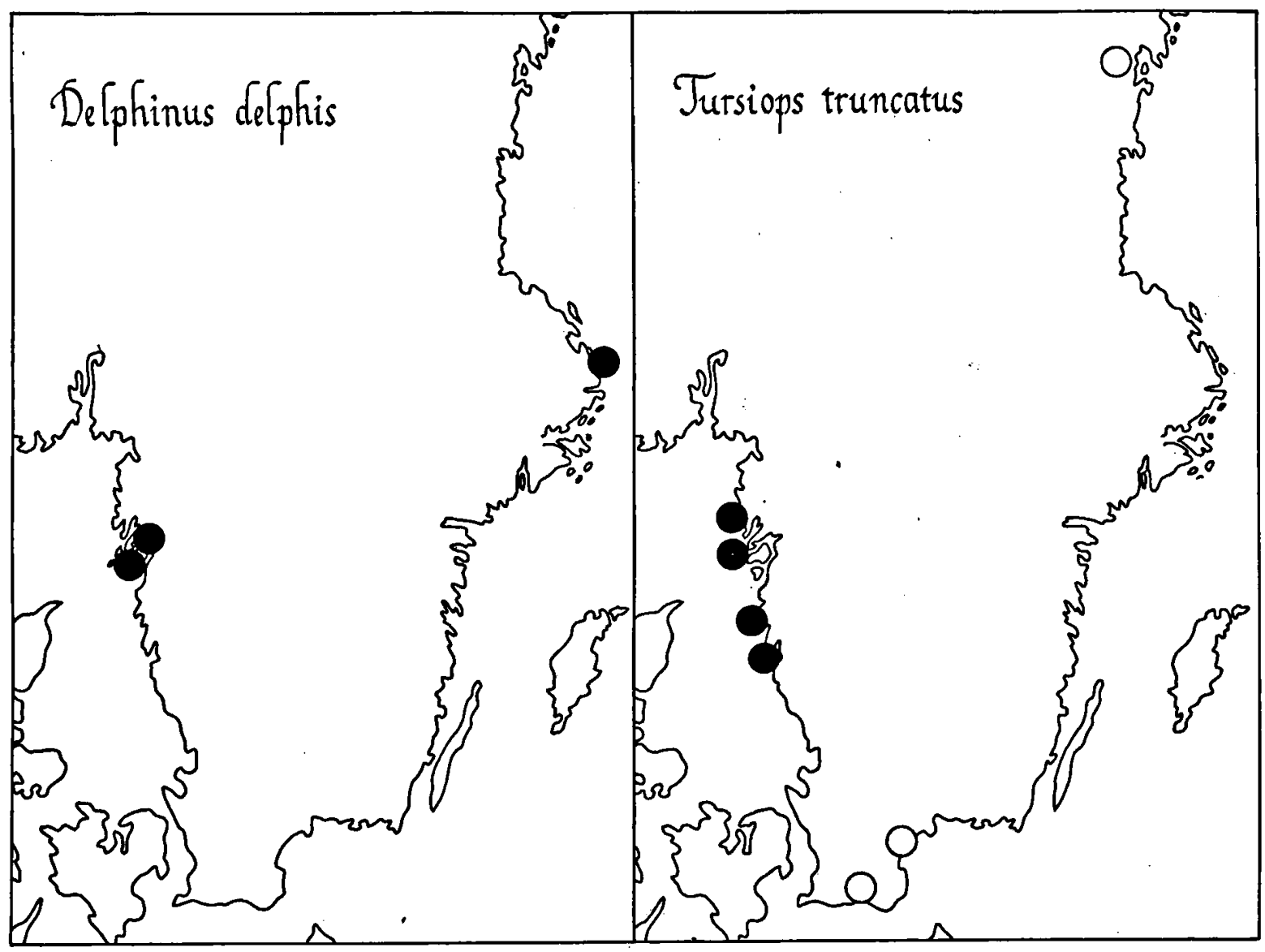

Abb. 4. Fundorte der ïberwiegend fischfressenden Kosmopoliten: des gemeinen Delphines und des Großtümmlers. Delphinus delphis: Havstensfiärd, Boxvikskil, Marum.

Tursiops truncatus: Långö, Grötö, Hönö, Malö, Tosterup, Ahus, Ångermanland?

leicht gilt dasselbe auch für den Dögling von welchem ein Fund an der Skagerrakküste (Marieberg bei Uddevalla) offensichtlich der Wärmezeit zuzuschreiben ist.

In den hochwärmezeitlichen Schalenablagerungen auf Otterö (HessLand, 1943) hat man neben den dominierenden Resten des Schweinswales und des Langfinnendelphins auch Reste des Schwertwales und des Weiß-seitendelphins (beinahe vollständiges Skelett im Naturhist. Museum Göteborg: JÄGERSKIöLD, 1921). Es ist weniger wahrscheinlich, daß es sich hier nur um seltene Irrgäste handelt. Wahrscheinlich ist auch der gegenwärtig nordatlantische Weiß-seitendelphin damals ein viel häufiger Besucher des Skagerraks gewesen als das heute mit ihm der Fall ist.

Die Kleinwaljagd schein für die mesolithischen Bewohner der Westküste keine größere Bedeutung zu haben. Unter den ziemlich reichlichen Knochenresten der Rottjärnslid-Siedlung (HENRICr, 1935, und eigene
Untersuchungen) sind keine Zahnwalreste hervorgekommen. Dagegen hat man Schweinswal schon im Oresundgebiet gejagt, wo ich seine Reste in den Küchenabfällen der Arlöv-Siedlung festgestellt habe.

Von desto größerer Bedeutung ist dagegen der Kleinwalfang bei den neolithischen Küstenbewohnern der Westküste in der Spätwärmezeit gewesen. Reste des Schweinswales und Langfinnendelphins findet man in mehreren Fundstücken in den Küchenabfällen sowohl am Skagerrak bei Dafter (FrEdsjö, 1963) und Rörvik (HENRICI, 1936 und eigene Untersuchungen), wie auch an der Kattegattküste bei Gröninge. In der tief in das Land hineindringenden Bucht bei Gröninge scheint man Kleinwalen neben Schulen des Thunfisches und der Seehunde nachgestellt haben. Bei Rörvik hat man auch Schwertwal gejagt. Der Anzahl ihrer Funde nach im Verhältnis zu denjenigen des Schweinswales, muß man annehmen, da $\beta$ der Schwertwal und der Langfinnen- 


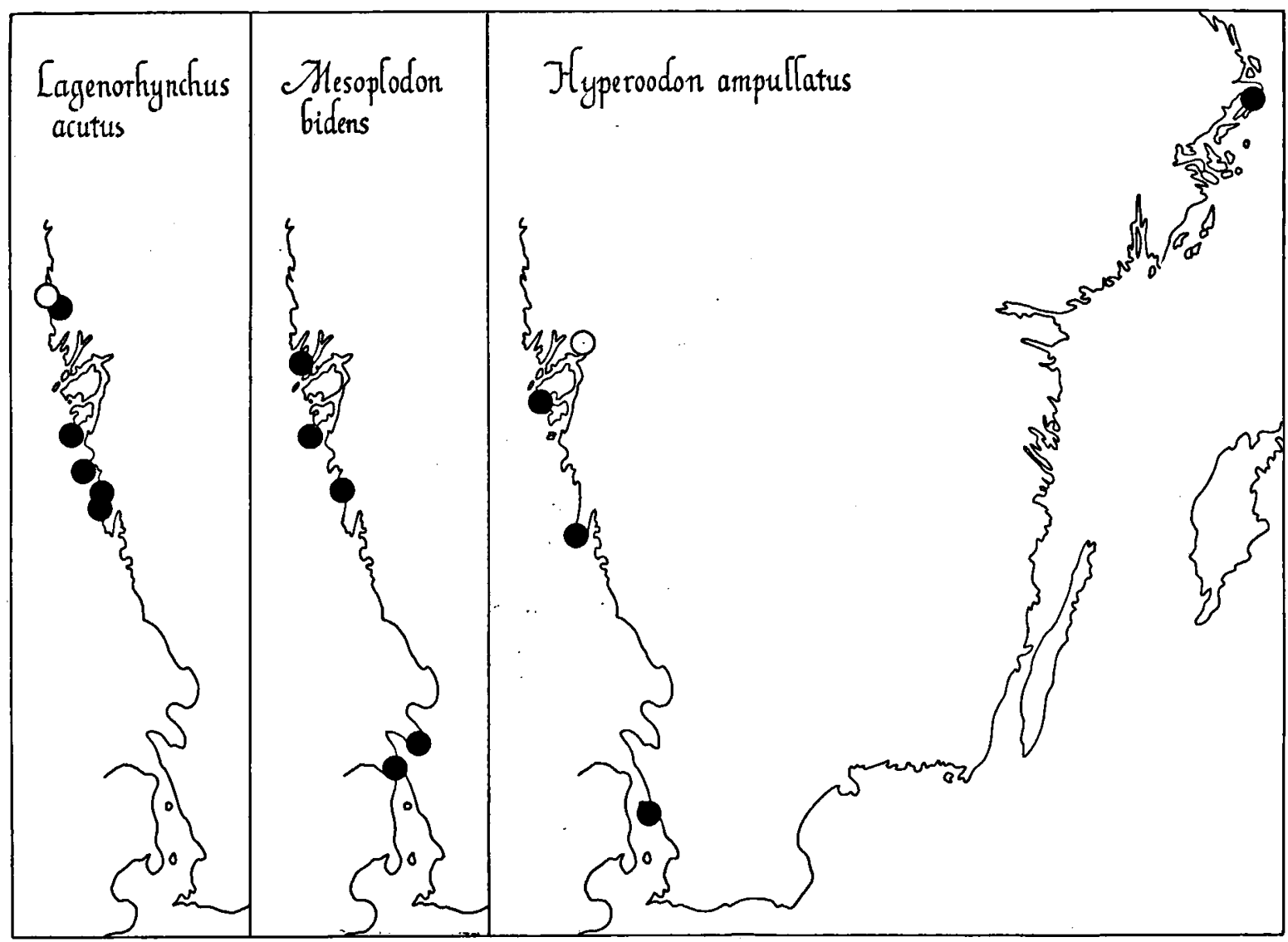

Abb. 5. Fundorte der nordatlantischen Irrgäste: des Weiß-seitendelphines, des Sowerby-zweizahnwales und des Döglings.

Lagenorhynchus acutus: Otterö, Fjällbacka, Marstrand, Hälsö, Bråttkärr, Kyvik.

Mesoplodon bidens: Saltö, Vannholm, Näset, Vegeholm, Hälsingborg.

Hyperoodon ampullatus: Marieberg, Mollö, Malö, Landskrona, Furusund.

delphin damals an der Westküste viel häufiger auftraten als heutzutage.

Ich möchte diesbezüglich auch auf die neusteinzeitlichen Felsenzeichnungen der naheliegenden norwegischen Skagerrakküste (Engelstadt, 1934; StiJPER, 1958) hinweisen, wo man neben Bildern von Elchen, Fischen, Fuchs und einer Riesenlumme (Riesenalke), auch zwei gute Abbildungen von größeren Zahnwalen vorfindet von welchen einer unverkenntliche Züge eines Lagenorhynchus, offensichtlich eines Langfinnendelphins, trägt. Die letztgenannte Art hat wahrscheinlich damals auch häufiger als jetzt die Südküste besucht und ist auch dort ein Jagdobjekt gewesen. Noch aus der Ende der Spätwärmezeit, aus den bronzezeitlichen Küchenabfällen bei Hötofta an der Südwestküste habe ich ein Rest von dieser Art vorgefunden. Vielleicht ist ein Langfinnendelphin auch in der wohlbekannten bronze- zeitlichen Grabkammer bei Kivik im Ostteil der Südküste dargestellt worden. Das stark schematisiert dargestelltes Tier scheint jedenfalls viel größer als ein Schweinswal zu sein.

Aus der Nachwärmezeit sind die prähistorischen Zahnwalfunde selten und begrenzen sich auf einige Schweinswalfunde in den jungeisenzeitlichen Küchenabfällen der Südküste. Daß man diese Art auch im Mittelalter im Öresundgebiet gejagt hat zeigen uns entsprechende Funde der Lahebiahöhle.

Merkwürdigerweise sind einzelne Reste des Schwertwales (Wirbeln und Zähne) wiederholt in den Kulturschichten der mittelalterlichen Städte des 11. bis 15. Jh. angetroffen worden. So in Lund, in den alten Kongahälla bei Kungälv und in Gamla Lödöse. Diese sind offensichtlich als von den Seeleuten mitgebrachten Curiosa oder Rohstoffe für Knochenschnitzerei aufzufassen und demzufolge für die lokale 


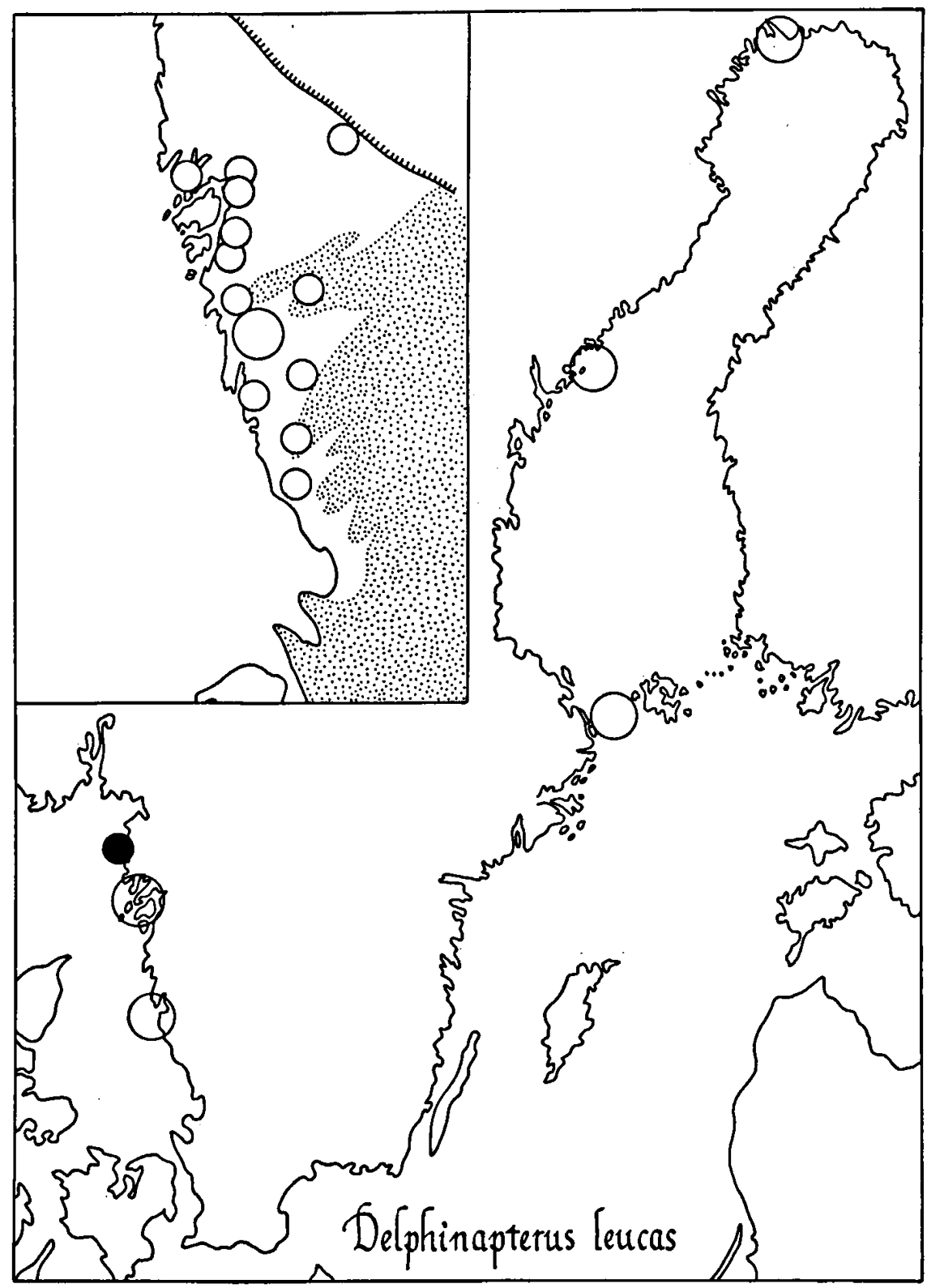

Abb. 6. Fundorte des Weißwales. Kleine Karte - die subfossilen Funde des vom spätglazialen und präborealen Meer ïberfluteten Teiles der Westküste. Die Eisrandlage oben mit Zackenlinie vermerkt, Land getïpfelt. Die Funde sind: Häggvall, Kuröd und Bräcke, Kilja-Moor (in der Nähe des Eisrandes), Pilgården, Grömosse, Agnesberg, Lerum, die Funde um und in Göteborg mit großem Kreis markiert (Slätta Damm, Kålltorp, Böö, Skår, Overås, Ȧnggården, Mölndal), Hulta, Olmanäs, Rolfstorp, Falkenberg.

Große Karte - rezentes Vorkommen. Tärnskär wo ein Weißwal im 1964 angeschwemmt wurde mit einem schwarzen Punkt markiert. Die Kreise bezeichnen die Gegenden wo man „weiße Wale im 1964 und 1841 (der Kreis höchst oben) beobachtet hat. 
Faunengeschichte von geringem Wert. Wenigstens vorläufig muß man dasselbe für einige andere Zahnwalfunde annehmen, die in den Hafenbereichen oder in Seefahrt- und Fischereistationen angetroffen worden sind.

Verzeichnis der Zahnwalfunde aus Schweden Die zahlreichen rezenten Funde der beiden für das Gebiet heimischen Arten, des Schweinswales und des Langfinnendelphins, sind nicht angeführt worden. Folgende Verkürzungen von Namen der Aufbewahrungsstätten des Belegemateriales sind angewendet: LUHM - Historisches Museum der Universität in Lund, LUZM - Zoologisches Museum der Universität in Lund, NMG - Naturhistorisches Museum in Göteborg, NRM - Naturhistorisches Reichsmuseum in Stockholm, UUZM - Zoologisches Museum der Universität in Uppsala.

Physeter macrocephalus Linnaeus, 1758, schwedisch Kaskelot.

\section{Rezente Funde}

1718, Ende XI - Bei Overö-Inseln an der Skagerrakküste von Bohuslän. Artbestimmt von Prof. L.

A. Jägerskiöld nach einer Reiseschilderung von C. Alstrin (im 1720). Näheres bei BernströM (1949).

\section{Erdfunde}

1871, kurz vor - Lillsved auf Värmdö, Uppland. MaLM (1871). Zahnfund im NRM. Historische Zeit?

1935 - Backa auf Skaftö, Bohuslän. JÄGERSKröLD (1936). Schulterblatt im NMG. Historische Zeit?

Hyperoodon ampullatus (Forster, 1770), schwedisch Dögling.

1823, IV - Bei Landskrona (Schonen) im Öresund. NILsson (1847, Hyperoodon borealis). Skelett im LUZM.

1879, - Bei Furusund (Uppland) an der Ostküste. JAPHA (1908, nach E. Lönnberg). 2 Tiere ( $q$ ad. und juv.) gestrandet. Skelette im NRM.

1902, 6.IX - An der Bohuslänsküste des Skagerraks? JAPHA (1908, nach E. LöNNBERG). 2 Tiere (6 und $6,5 \mathrm{~m}$ ) gestrandet. Nichts aufbewahrt.

1925 - Insel Malö (Halland) an der Kattegattküste. Teiles eines Skelettes im NMG.
1954, VIII/IX - An der Westseite der Insel Orust (Bohuslän). Kurz bevor freischwimmend bei Nösund und Tångesund beobachtet, am 2.IX tot auf die Insel Mollö angetrieben. Teile des Skelettes im NMG. In der Zeitung GöteborgsPosten von den 3.IX 1954 abgebildet (fälschlich als Grindwal bezeichnet).

\section{Subfossiler Fund}

1865, um - Marieberg bei Uddevalla (Bohuslän). Skelettreste der rechten Extremität im Ton. MaLM (1871). Uddevalla Museum.

Ziphius cavirostris Cuvier, 1823, schwedisch Småhuvudval.

\section{Rezente Funde}

1867, 22.IV - Bei Holma im Gullmarfjord (Bohuslän) an der Skagerrakküste. MALM (1871). Ein etwa 6,6 $\mathrm{m}$ langes $q$ tot angetrieben (unter Eisdecke erstocken?). Skelett im NMG.

1872, Anfang VIII - Bei Marstrand (Bohuslän) an der Skagerrakküste. LILLJEBorg (1874, Ziphius gervaisii). Ein über $6 \mathrm{~m}$ langes Tier (q?) gestrandet. Skelett im NRM.

Mesoplodon bidens (Sowerby, 1804), schwedisch Sowerbys näbbval.

\section{Rezente Funde}

1881, 9.XI - Insel Vannholm bei Marstrand (Bohuslän) an der Skagerrakküste. MaLM (1881, Micropteron bidens). Ein $4.5 \mathrm{~m}$ langes $\delta$ tot angetrieben. Skelett im NMG.

1885, 6.VIII - Insel Saltö bei Lysekil (Bohuslän) an der Skagerrakküste. Aurivillius (1887, Micropteron bidens). Etwa $4 \mathrm{~m}$ langes $\hat{o}$ juv. gestrandet und getötet. Skelett im NRM.

1938, 8.IX - Bei Vegeholm (Schonen) in der Skälderbucht des Kattegatts. BerLiv (1941). Ein 4,5 m langes $\delta$ tot angetrieben. Skelett im LUZM. 1950, 3.IX - Hälsingborg (Schonen) im Oresund. BERLIN (1951). Ein 4,47 $\mathrm{m}$ langes $\delta$ auf einer Sandbank gestrandet und getötet. Skelett im Malmö Museum.

1956, 7.IX - Näset im Askimfjord bei Göteborg (Västergötland) an der Kattegattküste. Ein 4,75 $\mathrm{m}$ langes $q$ ad. und ein $3,2 \mathrm{~m}$ langes $q$ juv. (Mutter und Kalb) getötet. Skelette und Gipsmodell des juv. Tieres im NMG. Im Zahnfleisch 
konnte der Verf. Reihen von rudimentären Zähne auspräparieren wonach folgende Zahnformeln sich ergaben:

\begin{tabular}{|lll|}
\hline & links: & rechts: \\
\hline 9 ad. & $\frac{0 . P}{1.16}$ & $\frac{0 . ?}{1.17}$ \\
& $\frac{0.6}{1.22}$ & $\frac{0.8}{1.23}$ \\
\hline
\end{tabular}

Schädelmaße (in $\mathrm{cm}$ ):
$4 \mathrm{~m}$ langer Weißwal tot angetrieben (Photobild in Göteborgs-Posten von den 18.IX 1964).

1964, Sommer - Kungsbackafjord (Halland) an der Kattegatküste. Ein weißer Wal von Fischern beobachtet (Konservator Björn Wennerberg mündl.).

1964 - Ostseeküste. Notizen in der Tagespresse: Anfang IX 3 weiße Wale im Schärenhof von Aland (Uppland), ein bei Örnsköldsvik (Ångermanland) und ein bei Prästskär in der Nähe von Bygdeå (Västerbotten) im Bottnischen Meerbusen beobachtet (Svenska Dagbladet von den 8.IX und 19.IX 1964).

\begin{tabular}{|c|c|c|c|}
\hline \multirow{2}{*}{ Fundort: } & \multirow{2}{*}{$\begin{array}{c}\text { Vannholm } \\
\hat{\delta} \mathrm{ad} .\end{array}$} & \multicolumn{2}{|c|}{ Näset } \\
\hline & & ㅇ ad. & q juv. \\
\hline Oberschädel & & & \\
\hline 1. Die größte Länge $\ldots \ldots \ldots \ldots \ldots \ldots \ldots \ldots \ldots$ & $73(+2 ?)$ & $78(+2 ?)$ & $46(+2 ?)$ \\
\hline$\ldots \ldots \ldots \ldots \ldots \ldots \ldots$ & 27,3 & 30,5 & 22,6 \\
\hline 3. Breite der Praemaxillaria hinter der Nasenöffnung & 12,1 & $13, \mathrm{C}$ & 10,3 \\
\hline Unterkiefer & & & \\
\hline 4. Die größte Länge & $63(+2 ?)$ & 69 & 40 \\
\hline 5. Die Länge der Symphyse $\ldots \ldots \ldots \ldots \ldots$ & $21(+2 ?)$ & 26,4 & 10,6 \\
\hline
\end{tabular}

Auffallenderweise sind die Funde dieser Art auf die Spätsommer und Herbstmonate beschränkt.

Delphinapterus leucas (Pallas, 1776), schwedisch Vitval.

\section{Rezente Funde}

1841, um - Bei Töre (Norrbotten) im Bottnischen Meerbusen. MaLM (1877). Ein kleiner weißer Wal beobachtet.

1964 - Skagerrakküste von Bohuslän. Notizen über weiße Wale (teilweise mit Photobilder) in der Tagespresse: Ende $\mathrm{V}$ ein weißer Wal im Hafen von Uddevalla, am 31.VIII wieder einer im Byfjord; Ende VIII/Anfang IX weiße Wale im Gullmarfjord, zwischen Skaftölandet und der Insel Orust, bei Svanesund und bei Ängö meist einzeln aber auch bis 3 Tiere gleichzeitig (Göteborgs-Posten von den 30.V und 8.IX 1964, Bohuslänningen von den 1.IX 1964).

1964, 17.IX - Insel Tärneskär bei Havstenssund (Bohuslän) an der Skagerrakküste. Ein etwa
Subfossile Funde (alle aus spätglazialen-präborealen Ablagerungen Westschwedens, alle im NMG aufbewhart; Nybelin, 1938, 1946).

1850 - Mölndal (Västergötland). MALM (1871, typus der Beluga catodon forma Mölndalensis Malm). Teile eines Skelettes im Ton. Wahrscheinlich nur ein altes, kleines $q$ der Art.

1860 - Overås in Göteborg. MALM (1871, Beluga catodon). Einzelfund (Wirbel von juv.) im schalenhaltigen Ton.

1868 - Grömosse bei Röröd, Jörlanda, Bohuslän. MALM (1871, Beluga catodon). Einzelfund (Schwanzwirbel von juv.).

1904, vor - Ölmanäs am Kungsbackafjord (Halland). Skelett im schalenhaltigen Ton.

1905 - Rolfstorp bei Varberg (Halland). Skelett im Ton.

1924 Kålltorp in Göteborg. Einzelfund (Wirbel) im Ton.

1925 - Stekens Ziegelei bei Agnesberg (Västergötland). Teile eines Skelettes im Ton.

1928 - Kuröd bei Uddevalla (Bohuslän). Abgerollter Axis aus der Schalenbank. 
1927-28 - Bräcke bei Uddevalla (Bohuslän). Skelettreste aus der Schalenbank. Ringström (1928).

1929 - Bei der Kirche von Lerum (Västergötland). Teile eines Skelettes im Ton.

1933 - Skår in Göteborg. Teile eines Skelettes (Rippen).

1933 - Böö in Göteborg. Teile eines Skelettes.

1934 - Pilgården, Norum (Bohuslän). Teile eines Skelettes (Schwanzwirbel) im Ton.

1936 - Ziegelei bei Falkenberg. Kopfskelett, etwa $10 \mathrm{~m}$ im Ton.

1937 - Anggården in Göteborg. Teile eines Skelettes im Ton.

1937 - Bräcke bei Uddevalla. Ein defekter Atlas aus der Schalenbank.

1938 - Häggvall, Lyse (Bohuslän). Skelett im Ton.
1943 - Kilja-Moor, Karaby (Västergötland). Einzelfund (Schwanzwirbel) im Ton.

1946 - Ziegelei in Hulta, Berghem (Västergötland). Skelett im Ton.

1947 - Kålltorp in Göteborg. Teile eines Skelettes im Ton.

1949 - Ziegelei in Hulta, Berghem (Västergötland). Skelett eines juv. im Ton.

1949 - Slätta Damm in Göteborg. Teile eines Skelettes im schalenhaltigen Ton.

Zwei Skelettfunde des Weißwales (von Agnesberg und Olmanäs) zeigen pathologische Deformierung der Wirbelkörper. Bei dem Schädel von Olmanäs ist der Schnauzenteil auffallenderweise nach rechts gebogen. Maße (in cm):

\begin{tabular}{|c|c|c|c|c|}
\hline Fundort: & Ölmanäs & Hägvall & Rolfstorp & $\begin{array}{c}\text { Falkenberg } \\
\text { juv. }\end{array}$ \\
\hline $\begin{array}{l}\text { Oberschädel } \\
\text { Größte Länge } \ldots \ldots \ldots \ldots \ldots \ldots \ldots \\
\text { Größte Breite } \ldots \ldots \ldots \ldots \ldots \ldots \\
\text { Kondylenbreite } \quad \ldots \ldots \ldots \ldots \ldots\end{array}$ & $\begin{array}{l}56.7 \\
26,1 \\
13\end{array}$ & $\begin{array}{l}51,5 \\
26,7 \\
13,2\end{array}$ & $\begin{array}{l}50,0 \\
27,8 \\
14,2\end{array}$ & $\begin{array}{l}34,8 \\
21,8 \\
11,2\end{array}$ \\
\hline $\begin{array}{l}\text { Atlas } \\
\text { Breite zwischen den Aussenkanten der } \\
\text { vord. Gelenkflächen } \quad \ldots \ldots \ldots \ldots \ldots\end{array}$ & 13,6 & 13,3 & 13,1 & - \\
\hline $\begin{array}{l}\text { Axis } \\
\text { Wie das Vorige } \quad \ldots \ldots \ldots \ldots \ldots\end{array}$ & 14,6 & 13,8 & 13,6 & - \\
\hline
\end{tabular}

Monodon monoceros Linnaeus, 1758, schwedisch Narval.

? - Byfjord bei Uddevalla (Bohuslän). Oberschädel. Vom Meeresboden aufgefischt. Uddevalla Museum. Historische Zeit?

? - Vapnö (Halland). Wirbel. NRM?

Phocaena phocoena (Linnaeus, 1758), schwedisch Tumlare.

\section{Subfossile Funde}

1874 - Kyvik (Halland) bei Göteborg. Skelett im schalenhaltigen Ton. NMG. Wärmezeit?

1916 - Gamlestaden in Göteborg. Skelettfund. NMG. Wärmezeit?

1933-1942 - Otterö (Bohuslän). Abgerollte Skelett- reste mehrerer Individuen aus der Schalenbank. NMG und Paläontologisches Institut der Universität in Uppsala. HessLand (1943). Atlantische Zeit.

1937 - Bräcke bei Uddevalla (Bohuslän). Abgerollte Skelettreste aus der Schalenbank. NMG. Präboreale Zeit.

1936 - Rörvik (Bohuslän). Henricr (1936). Eine Wirbel aus der Kulturschicht einer neolithischer Siedlung. NMG.

1934 - Fågelsten, Lindome (Halland). Schulterblatt, $3 \mathrm{~m}$ tief in der Erde. NMG.

1947 - Dafter Skee (Bohuslän). Eine Wirbel aus der Kulturschicht einer neolithischer Siedlung? NMG.

1949 - Dalköpinge bei Trelleborg (Schonen). Oberschädel im Dünensand. NMG. Subrezent? 
1949-51 - Valleberga (Schonen). LePiKsAAR (1961). Angeschnittene Wirbeln in der Kulturschicht einer jungeisenzeitlicher (Vendelzeit) Siedlung. LUHM.

1956 - Lahebia-Höhle am Kullaberg (Schonen). Wirbelkörperrest aus der Kulturschicht vom Mittelalter. LUHM.

1961 - Arlöv (Schonen). Knochenreste aus einer mesolithischen Kulturschicht. LUHM.

1961 - Gröninge (Halland). Schädelreste aus der Kulturschicht einer neolithischer Siedlung. Kungsbacka.

1964 - Hagestad (Schonen). Wirbelrest aus der Kulturschicht vom Mittelalter. LUHM.

Orcinus orca (Linnaeus, 1758), schwedisch Späck huggare.

\section{Rezente Funde}

1848, vor - Lysekil (Bohuslän) an der Skagerrakküste. MaLM (1871, Orca Eschrichtii). Schäde! (früher als ein Hackklotz gedient!) eines auffallend großen Tieres. NMG.

1820, um - Trelleborg (Schonen) an der Südküste. NiLsson (1820). Tot angetrieben. Schädel im LUZM?

1851, Ende III - Bei Ronneby (Blekinge) an der Südküste. NiLsson (1851). Ein über $7 \mathrm{~m}$ langes $\delta$ tot angetrieben. Skelett in LUZM.

1865, - Tot in der Ostsee nördlich Bornholm treibend angetroffen und von dort nach Gotland zum Skelettieren geführt. MALM (1871, Orca gladiator). Genauere Fundumstände bei LiLLjEBORG (1874). Skelett in UUZM.

1866, um - Åckerös zwischen Falkenberg und Morups Leuchtturm (Halland) an der Kattegattküste. MALM (1871, Orca minor). Ein etwa $6 \mathrm{~m}$ langes Tier tot angetrieben. Teile des Skelettes im NMG.

1868, 18.XII - Bei Lysekil (Bohuslän) an der Skagerrakküste. MALM (1871, Orca gladiator). Ein 3,7 $\mathrm{m}$ langes $\delta \hat{~ j u v . ~ g e s t r a n d e t ~ u n d ~ g e t o ̈ t e t . ~}$ Skelett im NMG.

1871, Anfang XII - An der Skagerrakküste von Bohuslän. Friesen (1872, Orca stenorhynchus). Ein etwa $3,5 \mathrm{~m}$ langes $o$ juv. tot und treibend angetroffen. Skelett im NRM.

1878, I - An der Skagerrakküste von Bohuslän. JAYHA (1908, nach einer Mitteilung von Prof. E. Lönıberg, Stockholm). Ein $q$ ad. erbeutet. Skelet im NRM.
1880, 13.V - Bei Balgö-Insel (Halland) an der Kattegattküste. Dickson (1883, Orca Eschrichtii). Ein + erbeutet. Skelett im NMG.

1893, Winter - Bei Varberg (Halland) an der Kattegattküste. JAPHA (1908, nach einer Mitteilung von Prof. L. A. Jägerskiöld, Göteborg). NMG.

1902, Sommer - Bei Kullen (Schonen) zwischen Kattegatt und Öresund. LöNNBERG (1903).

1913, 4.I - Vegeholm (Schonen) an der Kattegattküste. BerLin (1941). Ein juv. gestrandet. Schädel im LUZM.

1926, 13.IX - Vom Meeresboden bei Simrishamn (Schonen) an der Südküste aufgefischt. BERLIN (1941). Ein Schädel im LUZM.

1932 , - wie das Vorige.

1939 - Nordöstlich des Kulla-Leuchtturmes (Schonen) im Kattegatt. BerLIN (1941). Ein Schädel vom Meeresboden aufgefischt. Museum in Hälsingborg.

1942, 12.IV - Insel Brattö (Halland) an der Kattegattküste. NybeliN (1943). Ein 5,55 (?) m langes $q$, tot angetrieben. Ein Zahn im NMG.

1945, 1.IX - Frillesås (Halland) an der Kattegattküste. Nybelin (1946). Teile eines Skelettes im NMG.

1951, - Insel Vändelsö (Halland) an der Kattegattküste. Curry-Lindahl (1955).

Die Funde des Schwertwales scheinen sich auf alle Jahreszeiten zu verteilen. Auffallend viele stammen aus der Eisperiode des Jahres. Sowohl juvenile wie adulte Tiere sind angetroffen worden, jedoch keine trächtige Weibchen oder neulich geborene Jungtiere.

\section{Subfossile Funde}

1841, vor - Umgebung von Ivö (Schonen). MALM (1871, Orca gladiator). Einzelfund (Schwanzwirbel) im LUZM. Subrezent?

1849, - Bei Varberg (Halland). MaLm (1871, Orca minor). Teile eines Skelettes im Torfmoor. NMG.

1857 - Lund (Schonen). MaLm (1871, Orca gladiator). Erdfund im LUZM. Mittelalter?

19.Jh., Mitte - Ytterby (Bohuslän). MALM (1871, Orca gladiator). Erdfund an der Stelle der alten Kongahälla. Durchgebohrter Zahn im Histor. Mus. in Göteborg. Mittelalter.

19.Jh. - Ormatofta, südwestlich Kristianstadt (Schonen). Malm (1871, Orca Eschrichtii). Oberschädel. LUZM.

1871 - Castellgården in Ytterby bei Kungälv (Bohuslän). Erdfund (Wirbel) im NMG. Mittelalter.

1933 - Sotenkanal. Oberschädel gefunden bei den 
Kanalarbeiten im Schalenkiesschicht auf dem Ton. NMG. Wärmezeit?

1934 - Rödmyr-Moor bei Hälleviksstrand an der Insel Orust (Bohuslän). Teile eines Skelettes im NMG.

1936-1942 - Otterö-Insel bei Grebbestad (Bohuslän). Zähne und 2 Felsenbeine aus der Schalenbank. NMG und Paläont. Inst. der Universität in Uppsala (Hessland, 1943).

1936 - Rörvik (Bohuslän). Henrici (1936). Zähne in der Kulturschicht einer neolithischer Siedlung. NMG.

1963-1965 - Gamla Lödöse (Västergötland). LEPIKSAAR (1965). 2 angeschnittene Zähne aus der Kulturschicht des Mittelalters. NMG.

1962 - Rörö-Insel (Bohuslän) bei Göteborg. Ein Zahn aus dem Sand. NMG.

Pseudorca crassidens (Owen, 1846), schwedisch Halvspäckhuggare.

\section{Rezente Funde}

1861/62, Winter - Bei Limhamn (Schonen) an der Oresundküste. LILljEBong (1874). Ein Kadaver gestrandet.

1862, 31.V - An der Mündung des Sege-Flusses bei Malmö (Schonen) im Öresund. MaLm (1871) und LILlJEBorg (1874, nähere Fundumstände). Ein Kadaver (vielleicht das Vorige) angeschwemmt. Skelett im LUZM.

1862, im Herbst - Bei Torekov nahe Båstad (Schonen) an der Kattegattküste. MaLm (1871). Skelett im LUZM.

1862 - Bei Ystad (Schonen) an der Südküste. LiLlJEBorg (1874). Tot angetrieben. Teile des Skelettes im UUZM.

1862, Sommer - Bei Falkenberg (Halland) an der Kattegattküste. MALM (1871). Ein Kadaver angeschwemmt. Skelett ohne Schädel im NMG.

1862, um - Bei Falkenberg (Halland). Ein Schädel, das gut zum vorigen Skelett passt im Jahre 1938 dem NMG überliefert. NyBELIN (1939).

1920, um - Bei Varberg (Halland) an der Kattegattküste. Mathiasson (1960). Ein Wirbel (vom 1862?) im NMG.

$1934-5 \mathrm{~km}$ westlich Vinga (Västergötland) in Fischnetzen gefangen. JÄGERSKIÖLD (1935). Skelett und Gipsmodell im NMG. Ein $\widehat{\partial}, 3,69 \mathrm{~m}$ lang.

Das isolierte Vorkommen des letztgenannten Tieres ist merkwürdig. Vielleicht hat es sich von derselber
Herde verirrt von welcher mehrere im gleichen Jahr an der Südküste von Wales gestrandet haben (SourHERN, 1964). Das Tier ist als ziemlich kleines zu bezeichnen (die größte Länge des Oberschädels mißt nur 60,5 und die größte Breite $35,2 \mathrm{~cm}$ ).

\section{Subfossile Reste}

Der von Hessland (1943) aus Otterö erwähnte Zahn ist sicherlich fehlbestimmt geworden.

Globicephala melaena (Traill, 1809), schwedisch Grindval.

\section{Rezente Funde}

1861, 3.XI - An der Westseite der Insel Tjörn (Bohuslän) an der Skagerrakküste. MALM (1871, Globiocephalus svineval). Schädel im NMG.

1884, 18.VI - Bei Björlanda, Hisingen (Bohuslän) an der Skagerrakküste. Stuxberg (1885). Skelett im NMG.

Diese seltene Irrgäste sind beide adulte Tiere, jedoch verschiedentlicher Größe (die größte Länge des Oberschädels ist 68,3 und $57,5 \mathrm{~cm}$, die größte Breite 49,6 und $39,4 \mathrm{~cm}$ ).

\section{Erdfunde}

1945 - Värbyfjord bei Grundsund (Bohuslän). NYBELIN (1946). Eine korrodierte Lendenwirbel vom Meeresboden aufgebaggert. NMG. Historische Zeit?

Grampus griseus (Cuvier, 1812), schwedisch Rissos delfin.

\section{Rezenter Fund}

1927, 18.VI - Bei Gåsö (Bohuslän) an der Skagerrakküste. LöNNBERG (1927). Ein 3,46 m langes ㅇ tot angetrieben. Skelett im NRM.

Tursiops truncatus (Montagu, 1821), schwedisch Öresvin.

\section{Rezente Funde}

1853, um - An der Skagerrakküste von Bohuslän. LinzjeBorg (1862, Delphinus tursio). Schädel im LUZM.

1897, 17.XI - Insel Hönö (Bohuslän) bei Göteborg an der Skagerrakküste. JAPHA (1908, nach Prof. 
L. A. Jägerskiöld, Göteborg?). Schädel im NMG.

1937, VIII - Insel Långö bei Bovallsstrand (Bohuslän) an der Skagerrakküste. NxBELIN (1939). Eine Schädeldecke (offensichtlich längere Zeit am Meeresboden gelegen) im NMG.

1944, 18.VIII - Insel Grötö südlich Gåsö (Bohuslän) an der Skagerrakküste. Zuerst als Lagenorhynchus albirostris registriert. Teile eines Skelettes im NMG.

1957 - Insel Malö (Halland) an der Kattegattküste. Ein Kadaver angeschwemmt. Schädel im NMG.

Alle sind erwachsene Tiere variabler Größe gewesen (die größte Länge des Oberschädels von Grötö mißt 49,3, die entsprechende des Hönö-Fundes 50,5 und von Malö $56,8 \mathrm{~cm})$.

\section{Erdfunde}

19.Jh. - Bei Tosterup, östlich von Ystad (Schonen). MaLm (1871). Näheres über Fundlokal (nach einer Mitteilung von Prof. S. Nilsson) bei Lilljebong (1874). Schädel aus einem Torfmoor. (Nach MaLM (1877) rezent und von Kåseberga um 1854). LUZM.

19.Jh. - Bei Åhus. LilljeBorg (1874). Oberschädelfragment aus Dünensand. Subrezent? NRM.

? - Angermanland. Exman (1949). Aus litorinazeitlichen Ablagerungen.

Delphinus delphis Linnaeus, 1758, schwedisch Springare.

\section{Rezente Funde}

1848 - Skagerrakküste von Bohuslän. MALM (1871). Schädel im NRM.

1943, 19.IX - Boxvikskil-Bucht auf der Insel Orust (Bohuslän) an der Skagerrakküste. NybELiN (1943). Ein 2,06 m langes 9 gestrandet. Skelett und Gipsmodell im NMG.

1956, VIII - Marum (Uppland) an der Ostküste am Eingang zum Bottnischen Meerbusen. Ein 2,1 m langes Tier gestrandet. NRM.

1964, 1.IX - Havstensfjärd bei Utby, Herrestad (Bohuslän) an der Skagerrakküste. Ein $\hat{\delta}$ tot angeschwemmt. Skelett im NMG.

Die größte Länge des Oberschädels von Boxvikskil mißt 43,2 und derjenigen von Havstensfjärd $45,0 \mathrm{~cm}$.

Lagenorhynchus acutus 'Gray, 1828), schwedisch Vitslaing.

\section{Rezente Funde}

19.Jh. - Prof. S. Nizsson (1847) will sich erinnern, daß ein Wal dieser Art (Delphinus leucopleurus) bei Marstrand (Bohuslän) an der Skagerrakküste erbeutet wäre.?

1870, 1.X - Bei Fjällbacka (Bohuslän) an der Skagerrakküste. LILLJEBORG (1874). Skelett im UUZM.

1907, 14.VIII - Bei Orvik in der Nähe von Kyvik (Halland) an der Kattegattküste. JÄGERSKIöLD (1908). Skelett im NMG.

1917, Sommer - Insel Lindholm westlich Hälsö (Bohuslän). JÄGERSKıöLD (1917). Oberschädel im NMG.

1921, 18.XII - Bei Bråttkärr (Västergötland) bei Göteborg. JÄGERSKIöLD (1923). 2 Tiere gestrandet. Skelett eines $\delta$ im NMG, eines anderes Tieres im NRM.

Mit Ausnahme des Hälsö-Fundes, das juvenile Züge aufweist (die größte Breite des Oberschädels ist nur $19,4 \mathrm{~cm}$, die größte Breite der Nasenöffnung 4,5 cm) sind die übrigen im NMG adulte Tiere (die größte Länge des Oberschädels von Örvik mißt $37,7 \mathrm{~cm}$, derjenigen von Bråttkärr $40,3 \mathrm{~cm}$; die entsprechenden größten Breiten sind 20,5 und 22,5 cm, die Breiten der Nasenöffnung 5,4 und $5,8 \mathrm{~cm}$ ).

\section{Subfossile Funde}

1920 - Insel Otterö bei Grebbestad (Bohuslän). JäGERSKröLD (1921). Beinahe vollständiges Skelett aus der Schalenbank im NMG. Atlantische Zeit. Die größte Länge des Oberschädels ist $39,3 \mathrm{~cm}$, die größte Breite $22,6 \mathrm{~cm}$.

Lagenorhynchus albirostris Gray, 1846, schwedisch Vitnos(ing).

\section{Subfossile Funde}

1920-42 - Insel Otterö bei Grebbestad (Bohuslän). JÄGERSKröLD (1921). Abgerollte Skelettreste aus der Schalenbank im NMG. Atlantische Zeit. Breite der Nasenöffnung beim Oberschädelrest $8,0 \mathrm{~cm}$.

1931 - Ramholm bei Skaftö (Bohuslän). Brustbein im NMG.

1936 - Rörvik (Bohuslän). HenRIcI (1936). Skelettteile in der Kulturschicht einer neolithischer Siedlung. NMG.

1947-1948 - Dafter (Bohuslän). Fredsjö (1963). Skelettreste von wenigstens 3 Individuen aus der Kulturschicht einer neolithischen Siedlung? 
NMG. Breite zwischen Außenkanten der vorderen Gelenkflächen bei 3 Atlas mißt 9,8 , 10,4 und $10,8 \mathrm{~cm}$.

1952 - Nedre Kuröd bei Uddevalla (Bohuslän). Eine Lendenwirbel aus der Schalenbank. NMG.
1961 - Gröninge (Halland). Fragment eines Schläfenbeines in der Kulturschicht einer neolithischer Siedlung. Kungsbacka.

1965 - Hötofta (Schonen). Eine Wirbel in bronzezeitlicher Kulturschicht. LUHM.

\section{LITERATUR}

Aurivillius, C. W. S., 1887: Osteologie und äuBere Erscheinung des Wals Sowerby's (Micropteron bidens Sow.); K. Sv. Vet. Akad. Handl. 11.

BERLIN, H., 1941: Några sydsvenska valfynd från senare tid; Fauna och Flora.

- 1951: Ett nytt fynd av Sowerbys näbbval, Mesoplodon bidens (Sowerby), vid skånska kusten; Fauna och Flora.

BernströM, J., 1949: Om en kaskelot, Physeter catodon Linné, strandad i Bohuslän 1718. Fauna och Flora.

BృöRsıö, N., 1953: Bohussunden och den senglaciala landhöjningen. Göteborgs K. Vet.- och Vitterh. Samhälles Handl., sjätte följden, Ser. B, 6, No. 8.

Curry-Lindahl, K., 1955: Djuren i färg. DäggdjurenKräldjuren-Groddjuren. Stockholm.

Dickson, O., 1883: Catalogue of Collections from the Gothenburg Museum. Great International Fisheries Exhibition. VI. Skeletons of Dolphins and Whales. London.

Ekman, S., 1949: Valarna; Notini, G. \& Haglund, B.: Svenska Djur. Däggdjuren. Stockholm.

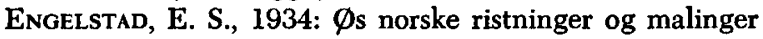
av den arktiske gruppe. Oslo.

FredsJö, Å., 1963: En stenåldersboplats vid Dafter i Skee socken; Bohusläns Hembygds Förbunds Årskrift.

FrIESEN, O. voN, 1872: Anteckningar angående en Orca, som anträffats vid Bohuslänska kusten i December 1871; Öfv. K. Vet. Akad. Förhandl. 29. Årg., No. 10.

GREvÉ, K., 1909: Säugetiere Kur- Liv- Estlands. Riga.

Hanström, B., 1950: Däggdjur, fåglar, kräldjur och groddjur i det Halland Naturskyddsföreningen tillhörande hedreservatet Steninge. Fauna och Flora.

HeNRICI, P., 1935: Benfynd från boplatsen på Rotekärrslid; Göteborgs och Bohusläns Fornminnesför. Tidskr.

- 1936: Benfynd från boplatsen vid Rörvik; Göteborgs och Bohusläns Fornminnesför. Tidskr.

Hessland, I., 1943: Marine Schalenablagerungen NordBohusläns; Bull. of Geol. Instit. of Upsala.

JAPHA, A., 1908: Zusammenstellung der in des Ostsee bisher beobachteten Wale; Schrift. d. Physik.-ökonom. Ges. zu Königsberg.

JÄGERSKIÖLD, L. A., 1908-1937: Naturhistoriska Museet. Zoologiska avdelning. Berättelse för 1908-1936; Göteborgs Musei Årstryck.

LePIKSAAR, J., 1961: Tierreste der Siedlungen von Valleberga und Rinkaby; M. Strömberg: Untersuchungen zur jüngeren Eisenzeit in Schonen. Acta Archaeologica Lundensia. Ser. in $4^{\circ}$, No. 4.

- 1965: Djurrester från Gamla Lödöse; Göteborgs Naturhistoriska Museum, Årstryck.

LILLJEBORG, W., 1862: Öfversigt af de inom Skandinavien
(Sverige och Norrige) anträffade Hvalartade Däggdjur (Cetacea); Upsala Univ. Årsskr. 1861-62.

- 1874: Sveriges och Norges Ryggradsdjur. I, Dägddjuren. Upsala.

LinNaEUS, C., 1751: Skånska resa . . . Förrättad år 1749. Stockholm.

LÖNNBERG, E., 1903: Undersökningar rårande Skeldervikens och angränsande Kattegat-omrädes djurlif; Meddel. från Kongl. Landtbruksstyrelsen No. 2 år 1902 (No. 80). Uppsala.

- 1927: Rissos Delfin, Grampus griseus, strandad i Bohuslän. Fauna och Flora.

Malm, A. W., 1871: Hvaldur i Sveriges museer år 1869. K. Sv. Vet. Akad. Handl. Ny Följd 9.

- 1877: Göteborgs och Bohusläns Fauna, Ryggradsdjuren. Göteborg.

Malm, A. H., 1881: Om Micropteron bidens (Sow.) Malm $\hat{\delta}$, Smådögling, funnen nära Marstrand den 30 , Oktober 1881. Göteborgs Naturhist. Mus. III Årsskr.

Mathiasson, S., 1960: Faunistiskt nytt från Göteborgs Naturhistoriska Museum 1959. Göteborgs Naturhistoriska Museum, Årstryck.

Nilsson, S., 1820: Skandinavisk Fauna. I Auflage. Lund. - 1847: Skandinavisk Fauna. II Auflage. Lund.

- 1855: Om den tandhval (Delphinus orca) som sistlidne vår strandat vid kusten af Blekinge. Förhandl. skand. Naturforsk. 6 möte 1851. Stockholm.

NybeLIN, O., 1938-1959: Naturhistoriska Museet. Berättelse för 1937-1959; Göteborgs Musei Årstryck.

- 1938: Forntida djur från det nutida Göteborg. Tidskr. för Göteborgs stads tjänstemän No. 10.

- 1944: Två sällsyntheter och en nyhet för den svenska havsvertebratfaunan. Fauna och Flora.

- 1946: Forntida djur från det nutida Västergötland. Lidköping.

ODHNER, N., 1930: Skalbankarna vid Uddevalla; Naturens Liv, H. 10 och 11 . Stockholm.

Ringström, T. J., 1928: Vertebratfynd i finiglaciala skalbankar vid Uddevalla. Meddel. från Göteborgs Musei Zoologiska avdelning 45.

SLIJPER, E. J., 1958: Walvissen. Amsterdam.

SoutherN, H. N., 1964: The Handbook of British Mammals. Oxford.

STUXBERG, A., 1885: Berättelser afgifna af Intendenterna vid Göteborgs Museum, rörande de derstädes befintliga samlingar, för år 1885.

SvÄrdSON, G., 1957: Goda laxår - och dåliga. Stockholm. Winge, H., 1904: Om jordfundne Pattedyr fra Danmark. Vidensk. Meddel. fra den naturh. Foren. i Kjøbenhavn. 